Article

\title{
Effects of Grain Size on Ultrasonic Attenuation in Type 316L Stainless Steel
}

\author{
Tao Wan *, Takashi Naoe (D), Takashi Wakui, Masatoshi Futakawa, Hironari Obayashi \\ and Toshinobu Sasa (D) \\ J-PARC Center, Japan Atomic Energy Agency, 2-4 Shirakata, Tokai-mura, Ibaraki 319-1195, Japan; \\ naoe.takashi@jaea.go.jp (T.N.); wakui.takashi@jaea.go.jp (T.W.); futakawa.masatoshi@jaea.go.jp (M.F.); \\ obayashi.hironari@jaea.go.jp (H.O.); sasa.toshinobu@jaea.go.jp (T.S.) \\ * Correspondence: wan.tao@jaea.go.jp; Tel.: +81-029-282-6948
}

Received: 30 May 2017; Accepted: 30 June 2017; Published: 5 July 2017

\begin{abstract}
A lead bismuth eutectic (LBE) spallation target will be installed in the Target Test Facility (TEF-T) in the Japan Proton Accelerator Research Complex (J-PARC). The spallation target vessel filled with LBE is made of type 316 stainless steel. However, various damages, such as erosion/corrosion damage and liquid metal embrittlement caused by contact with flowing LBE at high temperature, and irradiation hardening caused by protons and neutrons, may be inflicted on the target vessel, which will deteriorate the steel and might break the vessel. To monitor the target vessel for prevention of an accident, an ultrasonic technique has been proposed to establish off-line evaluation for estimating vessel material status during the target maintenance period. Basic R\&D must be carried out to clarify the dependency of ultrasonic wave propagation behavior on material microstructures and obtain fundamental knowledge. As a first step, ultrasonic waves scattered by the grains of type 316L stainless steel are investigated using new experimental and numerical approaches in the present study. The results show that the grain size can be evaluated exactly and quantitatively by calculating the attenuation coefficient of the ultrasonic waves scattered by the grains. The results also show that the scattering regimes of ultrasonic waves depend heavily on the ratio of wavelength to average grain size, and are dominated by grains of extraordinarily large size along the wave propagation path.
\end{abstract}

Keywords: type 316L SS; grain size; ultrasonic waves; scattering; attenuation coefficient

\section{Introduction}

The management of high-level radioactive wastes (HLW) is a critical and challenging issue worldwide. To effectively and efficiently transmute the minor actinides included in HLW, the Japan Atomic Energy Agency has proposed an accelerator-driven system (ADS) for nuclear transmutation, which is equipped with a lead-bismuth eutectic (LBE) target/coolant system and an LBE enclosure vessel $[1,2]$. To solve the basic technical issues related to the design of ADS, such as LBE flow behavior and ADS structural material behavior under high temperature, intense irradiation, and LBE flow, the construction of the Transmutation Experimental Facility (TEF), including an ADS Target Test Facility (TEF-T), has been proposed within the framework of the Japan Proton Accelerator Research Complex (J-PARC) project [3,4]. In TEF-T, an LBE spallation target will be installed and bombarded with pulsed proton beams ( $250 \mathrm{~kW}, 400 \mathrm{MeV}, 25 \mathrm{~Hz}, 0.5 \mathrm{~ms}$ pulse duration). Figure 1 shows a schematic drawing of the configuration of a half-LBE spallation target. The LBE vessel and the beam window (BW) will be made of $2 \mathrm{~mm}$-thick type 316L stainless steel for the first-stage operation of the TEF-T LBE spallation target. 


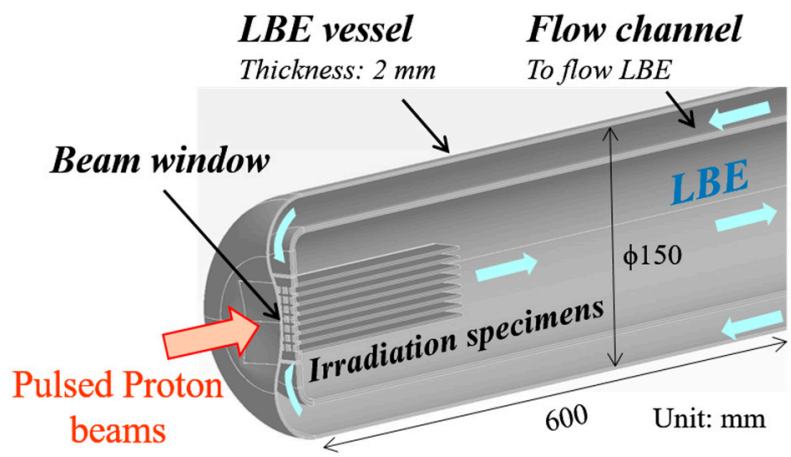

Figure 1. Schematic drawing of the configuration of a half-lead-bismuth eutectic (LBE) spallation target.

The LBE vessel, especially the BW, is loaded with various massive stresses [5], including the static pressure induced by LBE flow, thermal stress due to proton beam injection, and dynamic stress due to pressure waves. In addition to the high stress loadings, the LBE vessel should withstand many types of damages under rigorous service conditions as well; such as erosion/corrosion damage [6] and liquid metal embrittlement [7] caused by contact with flowing LBE at high service temperatures, and irradiation hardening due to protons and neutrons [8]. Those damages would significantly affect not only the surface morphology of the vessel, but also the microstructure of the vessel material, such as grain structure and dislocation density. Deterioration of the material will exacerbate its mechanical properties and will eventually lead to failure of the LBE vessel. Consequently, highly radioactive LBE will leak from the failed vessel and risk the environment.

To prevent the occurrence of an accident, exact evaluation of the vessel status during target operation and maintenance period is desired. For in-situ evaluation, a laser Doppler vibrometer technique, developed by the researchers from the mercury spallation target of the Japan Spallation Neutron Source [9,10], has been proposed to monitor the target based on its vibration behavior. As for off-line evaluation, the ultrasonic technique is a promising candidate for estimating the vessel material status owing to the following reasons:

(1) The space available for installation of the evaluation system is very restricted, and intense irradiation is anticipated in this space owing to protons and neutrons; moreover, a detection depth of several millimeters is required. These characteristics limit the applications of several typical non-destructive evaluation techniques, such as X-ray and eddy current to evaluate the vessel material.

(2) In general, the ultrasonic system is simple and compact enough to be integrated into the spallation target system, and it can be operated in the high-radiation environment.

(3) Information about the through thickness of the LBE vessel can be provided by ultrasonic waves propagating in the thickness direction, which is vital for material evaluation.

To establish the ultrasonic technique for vessel material evaluation, the correlation between the material status and the ultrasonic wave propagation behavior should be fully understood, and based on this correlation, a database can possibly be established in the future. Among classical ultrasonic methods, the ultrasonic attenuation method is considered very sensitive to changes in material microstructures [11,12].

As the first in a series of basic research steps to develop the ultrasonic technique, the present study systematically investigated the effects of grain size of type 316L stainless steel on ultrasonic wave attenuation through experiments and numerical simulations. The experiments provide an exact way to quantitatively evaluate the grain size through ultrasonic attenuation, while the numerical simulations constitute a new approach to modeling the interaction between grains and ultrasonic wave scattering in a material. The effects of grain size distribution, especially the influences of grains with extraordinary sizes, were discussed as well. 


\section{Theory of Ultrasonic Wave Attenuation}

The energy loss of ultrasonic waves in polycrystalline metals can principally be caused by the spreading, scattering and absorption of ultrasonic waves [13]. The spreading of cylindrical ultrasonic waves in material is expressed as follows:

$$
A_{D} \propto A_{0} R^{-\frac{1}{2}}
$$

where $A_{D}$ is the amplitude of ultrasonic waves at a position due to dispersion, $A_{0}$ is the amplitude of the source ultrasonic waves, and $R$ is the propagation distance.

The attenuation caused by scattering and absorption can be expressed by the following equations [14]:

$$
A_{R}=A_{0} \cdot e^{-\alpha \cdot t},
$$

where $A_{R}$ is the amplitude of the received ultrasonic waves, $\alpha$ is the attenuation coefficient, and $t$ is the propagation time. The attenuation coefficient can be divided into several parts according to various attenuation mechanisms, which can be expressed as [15]:

$$
\alpha=\alpha_{S}+\alpha_{A},
$$

where $\alpha_{S}$ is the attenuation coefficient due to scattering, and $\alpha_{A}$ is the attenuation coefficient due to absorption of ultrasonic waves in the material. Normally, the attenuation coefficient due to absorption is very small and can be ignored.

The theory of wave scattering from the grains satisfies the following assumptions: the grain structure is cubic, the number of scatters is inversely proportional to mean grain volume, the variation of elastic modulus is far smaller than the mean modulus, and the alloy is single-phase and consists of equiaxed grains, a schematic drawing of which is shown in Figure 2. The attenuation coefficient due to scattering by grains is related closely to the ratio of the wavelength of ultrasonic waves, $\lambda$, and grain size, $D$. Basically, it can be divided into three regimes, which are summarized in Table 1 [16-18], where $V_{g}$ is the volume of grains; $f$ is the frequency of ultrasonic waves; and $K_{1}, K_{2}$, and $K_{3}$ are constants related to material property.

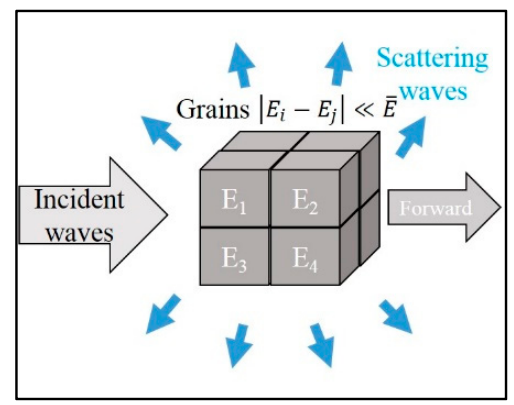

Figure 2. Schematic drawing of grain scattering.

Table 1. Classification of scattering type.

\begin{tabular}{ccc}
\hline Relationship & Scattering Regime & Attenuation Coefficient $\left(\alpha_{S}\right)$ \\
\hline$\lambda>2 \pi D$ & Rayleigh & $\alpha_{S}=K_{1} V_{g}{ }^{3} f^{4}$ \\
$\lambda \approx 2 \pi D$ & Stochastic & $\alpha_{S}=K_{2} D f^{2}$ \\
$\lambda<<D$ & Geometric & $\alpha_{S}=K_{3} D^{-1}$ \\
\hline
\end{tabular}

\section{Experiments}

\subsection{Material and Sepcimens}

Type 316L stainless steel, the candidate material of the TEF-T target vessel, was used in the experiment. To prepare the specimens of various grain sizes, heat treatment was first carried out under 
the following conditions: holding temperatures of 1173,1373 , and $1473 \mathrm{~K}$; holding time of $2 \mathrm{~h}$ in a vacuum; followed by natural cooling. Specimens measuring $20 \times 20 \times 2 \mathrm{~mm}^{3}$ were cut from the heat-treated samples for analyses.

Thereafter, the specimens were polished up to $0.06 \mu \mathrm{m}$ for metallurgical observation. After electrochemical etching in oxalic acid solution, the etched specimens were observed using a microscope. The mean grain sizes in 2D cross sections were measured by tracing grain boundaries through image analysis. The mean equivalent grain size was defined as the diameter of a circle equivalent to the area of a grain.

\subsection{Ultrasonic System}

The grain size of the specimens was evaluated using an ultrasonic system, as shown in Figure 3. Low-noise tone-burst sinusoidal waves were generated using a large-amplitude burst wave generator (RPR-4000, RITEC. Inc., Warwick, GA, USA). After amplifying using a high-power amplifier, the ultrasonic waves of frequency of $5 \mathrm{MHz}, 10 \mathrm{MHz}$, and $20 \mathrm{MHz}$ were sent out by focused transducers with focal lengths of $38 \mathrm{~mm}, 76 \mathrm{~mm}$, and $25 \mathrm{~mm}$, respectively. The ultrasonic waves reflected by the submerged specimens were received by the focused transducer again. The received waveforms were recorded automatically by the system by using an oscilloscope. Detailed information about the experimental setup can be found in [19].

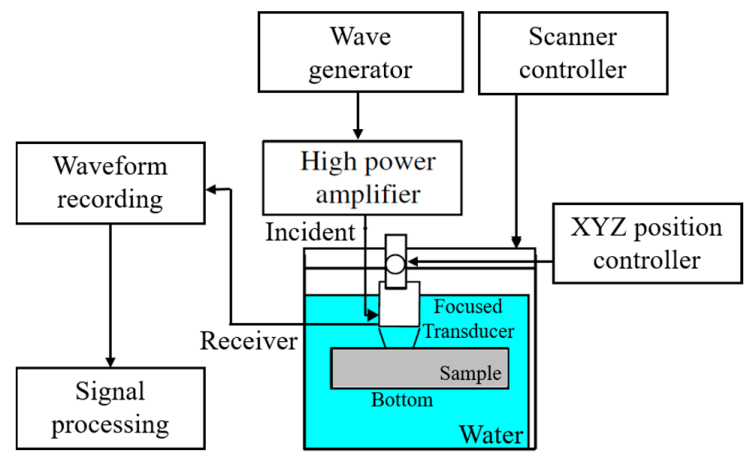

Figure 3. Schematic drawing of the ultrasonic system.

\section{Numerical Simulations}

\subsection{Time Step and Element Size}

To systematically investigate the dependency of ultrasonic wave propagation behavior on the grain size of the material, numerical simulations were carried out using the explicit analysis code of LS-DYNA [20]. The time step is an important parameter in the explicit analysis. If the time step is too long, the high-frequency components would be not resolved with sufficient accuracy. By contrast, if the steps are too short, the Personal Computer memory is affected and the calculation cost increases considerably. Therefore, one should always consider the balance, which is 20 points per cycle of the highest frequency component of interest [21]. The rule is expressed as follows:

$$
\Delta t<\frac{1}{20 f_{\max }}
$$

where $f_{\max }$ is the highest frequency of interest.

Furthermore, element size is another important parameter that should be taken into consideration to obtain accurate calculation results. It should be selected correctly so that the propagation waves are adequately resolved spatially. In general, at least 20 nodes per wavelength should be used [22]. This rule can be expressed as follows:

$$
S_{m}<\frac{\lambda_{\min }}{20}
$$


where $S_{m}$ is the mesh size and $\lambda_{\min }$ is the shortest wavelength of interest.

Here, the mesh size of models was selected as $2.5 \mu \mathrm{m}$ considering the memory size of the PC, and the time step was set to approximately $4 \times 10^{-11} \mathrm{~s}$.

In addition, the Courant number condition was satisfied to guarantee the accuracy and stability of the wave propagation solutions. The expression for Courant number, $C$, in a two-dimensional (2D) case is as follows:

$$
C=\frac{\Delta t V_{x}}{S_{m, x}}+\frac{\Delta t V_{y}}{S_{m, y}} \leq C_{\max }
$$

where $V_{x}$ and $V_{y}$ are the longitudinal ultrasonic wave propagation velocities in the $\mathrm{X}$ - and Y-directions, respectively, and $S_{m, x}$ and $S_{m, y}$ are the mesh sizes in the X-and Y-directions, respectively. Typically, $C_{\max }=1$ in an explicit calculation.

\subsection{Finite Element Models}

First, two types of 2D finite element models of different grain sizes were constructed to simulate square grains, as shown in Figure 4. The dimensions of each of the models were $3 \times 3 \mathrm{~mm}$. The sizes of square grains were $300 \mu \mathrm{m}$ (Figure 4a, Model A) and $150 \mu \mathrm{m}$ (Figure 4b, Model B). The models were meshed using shell elements with a plane strain type. The boundary conditions were non-reflective to avoid contamination by the ultrasonic waves reflected from the boundaries. The source node was assigned a sinusoidal profile load. The source position was set to $X=1.15 \mathrm{~mm}, Y=0.38 \mathrm{~mm}$, whereas the position of receiver was set to $X=1.15 \mathrm{~mm}, Y=2.99 \mathrm{~mm}$. To determine wave scattering due to grains, various values of Young's modulus $(E)$ were assigned to each grain. Generally, the distribution of $E$ obeys a normal distribution in metals [23], and the stand error is approximately $5 \%$ of the averaged value of $E$ [24]. In this study, the average value of the normally distributed $E$ was selected as $200 \mathrm{GPa}$, and the standard error was selected as $10 \mathrm{GPa}$, which can be expressed as follows:

$$
E \in N(200 \mathrm{GPa}, 10 \mathrm{GPa}) \text {. }
$$

In addition to the models with square grains, two other models were established to simulate grains of random size and geometry, as shown in Figure 5. The sizes and the geometries of the two models were replicas of the real grains of an Invar specimen treated at $1473 \mathrm{~K}$ (Model C) and as-received (Model D). The dimensions of each of the models were $2.4 \times 1.8 \mathrm{~mm}$. Model C contained 127 grains, while Model D contained 399 grains. The average grain sizes of the two models were approximately $193 \mu \mathrm{m}$ and $61 \mu \mathrm{m}$, respectively. The source position was set to $X=1.2 \mathrm{~mm}, \mathrm{Y}=0 \mathrm{~mm}$, whereas the receiver position was set to $X=1.2 \mathrm{~mm}, Y=1.75 \mathrm{~mm}$. The other conditions were the same as those described above.

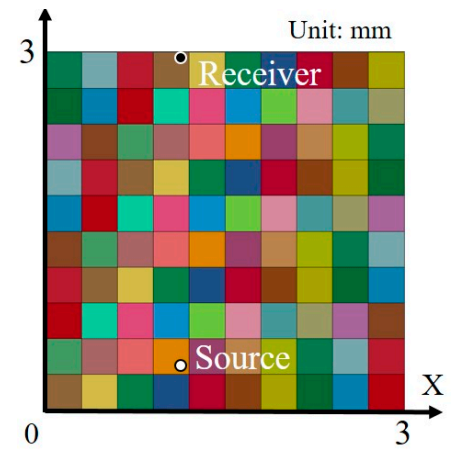

(a)

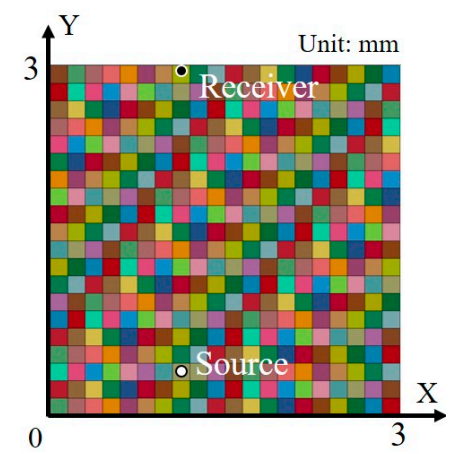

(b)

Figure 4. 2D model with square grains: (a) $300 \mu \mathrm{m}$ grain size (Model A); (b) $150 \mu \mathrm{m}$ grain size (Model B). 


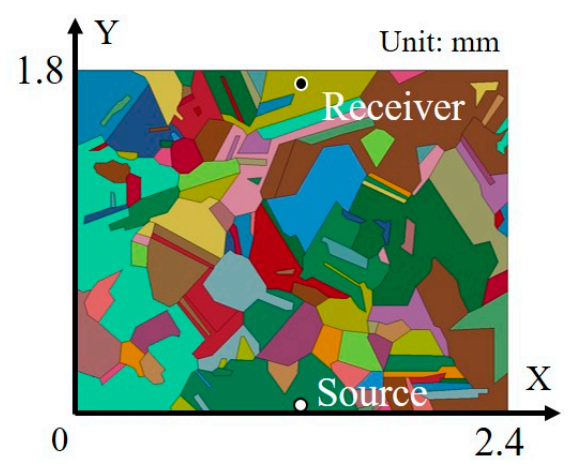

(a)

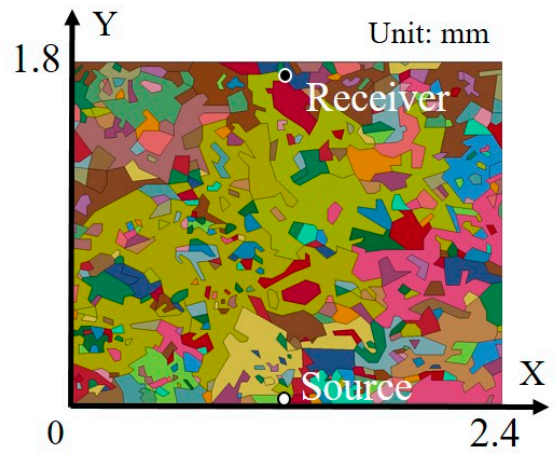

(b)

Figure 5. 2D model with random grains: (a) 127 random grains (Model C); (b) 399 random grains (Model D).

\section{Results}

\subsection{Grain Sizes}

Figure 6 shows the grain size as a function of the heat treatment temperature. Note that the grain size was obtained from a cross section in the thickness direction. It has been stated in Section 2 that the mean grain size is a parameter that affects ultrasonic attenuation. The mean grain size of the as-rolled type 316L SS sample is approximately $37 \mu \mathrm{m}$ at $298 \mathrm{~K}$. The grain size coarsened at higher temperatures. The mean grain size reached approximately $107 \mu \mathrm{m}$ when the temperature was increased to $1473 \mathrm{~K}$. However, the standard deviation of grain size tended to increase with increasing heat treatment temperature as well.

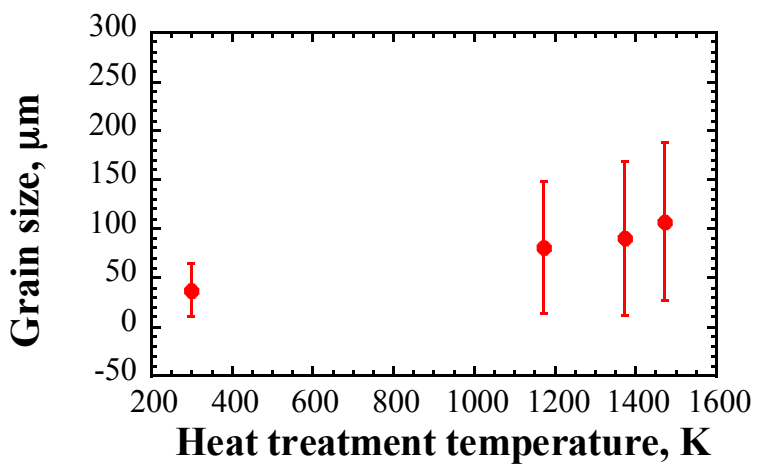

Figure 6. Grain size as a function of heat treatment temperature.

Figure 7 shows the histograms and the probability functions of the grain size distribution in the cross section of the specimens treated at various temperatures. It can be seen that the logarithmic grain size shows a normal distribution in the four cases; that is, the grain size distributions are log-normal, which is the typical even normal grain size distribution in metals. Moreover, the histograms show that the deviation of grain size distribution in the cases of specimens treated at $1173 \mathrm{~K}$ and $1373 \mathrm{~K}$ is attributed mainly to grains of considerably small sizes, while in case of the specimen treated at $1473 \mathrm{~K}$, it is caused mostly by very large grains. Actually, the sizes of approximately $0.5 \%$ of the grains exceed $500 \mu \mathrm{m}$ in the case of the specimen treated at $1473 \mathrm{~K}$, as shown in Figure $7 \mathrm{~d}$. Based on this result, we can attribute the large standard deviation in Figure 6 to two main reasons: (1) the grain size in the thickness direction is a log-normal distribution instead of a normal distribution; and (2) a few grains have very large or small sizes. 


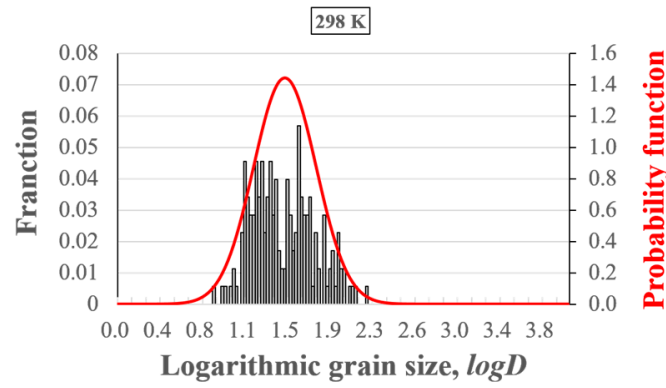

(a)

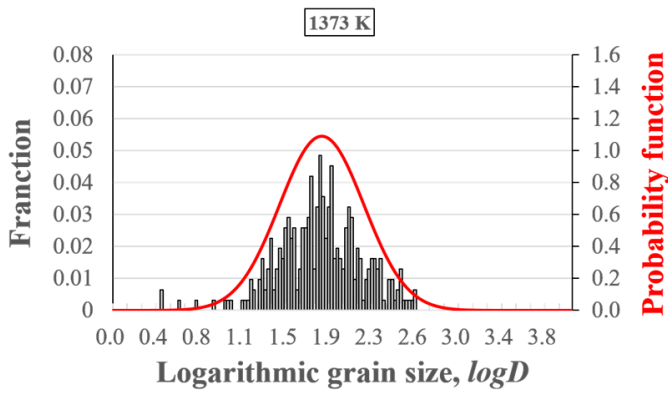

(c)

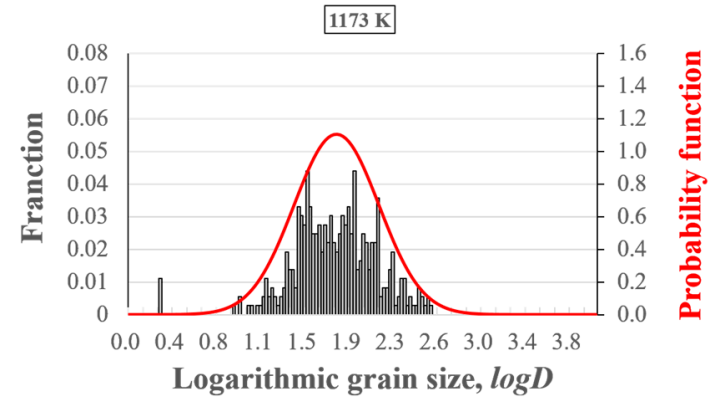

(b)

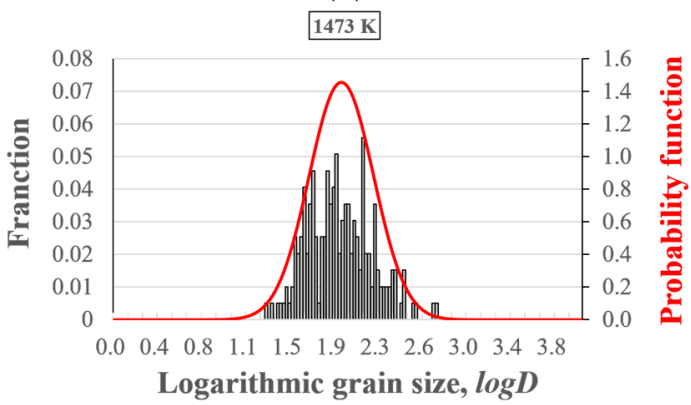

(d)

Figure 7. Histograms and probability functions of grain size distributions of specimens heat-treated at various temperatures: (a) $298 \mathrm{~K}$; (b) $1173 \mathrm{~K}$; (c) $1373 \mathrm{~K}$; (d) $1473 \mathrm{~K}$. The red solid lines show the normal distribution of the logarithmic grain size.

It should be noted that the grain sizes presented so far are obtained from only one cross section. To obtain the mean grain size in the specimen as precisely as possible, it is necessary to trace the grains in several cross sections instead of only one cross section, to calculate the average value of grain sizes and the corresponding standard deviation. However, such experiments are very time-consuming. Instead, a statistical analysis can be performed based on the data obtained from the current cross section to estimate the standard deviation of the average logarithmic grain size in the specimens. As described above, the logarithmic grain size distribution is normal. If it is assumed that each cross section in the specimen contains the same number of grains, which is reasonable, then the standard deviation of the average logarithmic grain size, $\sigma_{\overline{\bar{D}_{l}}}$, can be calculated using the following equation:

$$
\sigma_{\overline{D_{l}}}=\sigma_{D_{l}} / \sqrt{N}
$$

where, $\sigma_{\overline{D_{l}}}$ is the standard deviation of the averaged logarithmic grain size of the specimen; $\sigma_{D_{l}}$ is the standard deviation of the logarithmic grain size in the current cross section; $N$ is the number of grains in the current section; and subscripts $\overline{D_{l}}$ and $D_{l}$ denote the averaged logarithmic grain size and logarithmic grain size, respectively. It has been pointed out that $95 \%$ of the comparable averaged logarithmic grain size will fall within the range of $\overline{D_{l}} \pm 2 \sigma_{\overline{D_{l}}}$ [25].

Figure 8 shows the averaged logarithmic grain size as a function of heat treatment temperature. It should be mentioned that the error bar of the data represents $2 \sigma_{\overline{D_{l}}}$. A clear dependency of the averaged logarithmic grain size on the heat treatment temperature can be observed. The error bar is on a reasonable level. Therefore, the results in Figure 8 reflect that although the standard deviation of the grain size is large in Figure 6, the average grain size has a physical meaning. 


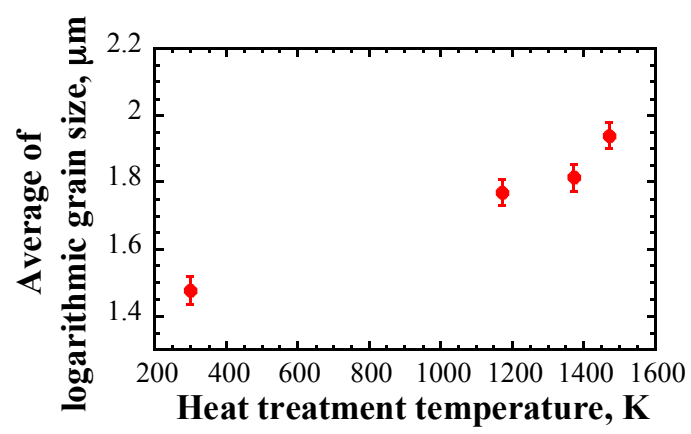

Figure 8. Average of logarithmic grain size as a function of heat treatment temperature; note that the error bar of data represents twice the standard deviation of the mean.

\subsection{Experimetnal Results}

Figure 9 shows two examples of the received waveforms from various samples (at $10 \mathrm{MHz}$ ). Assuming that the longitudinal ultrasonic wave propagation velocities in water and in 316L SS are $1.45 \mathrm{~mm} / \mu \mathrm{s}$ and $5.9 \mathrm{~mm} / \mu \mathrm{s}$, respectively, the signal marked $A_{u}$ is reflected from the upper surface of the sample (out of the range of display), while the signals marked $A_{1}, A_{2}$, and $A_{3}$ are back-wall waveforms reflected from the bottom side of the specimen. A schematic drawing of wave propagation is shown in Figure 10. A part of the ultrasonic waves reflected from the upper surface of the specimen and the other part keep propagating along the thickness direction of the specimen, and then travel between the upper and the bottom surfaces of the specimen.

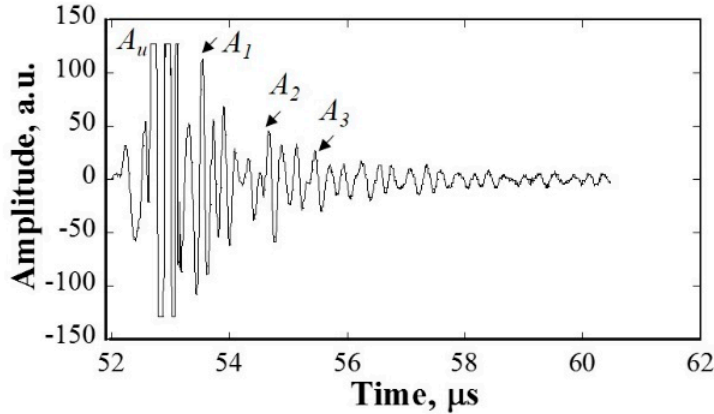

(a)

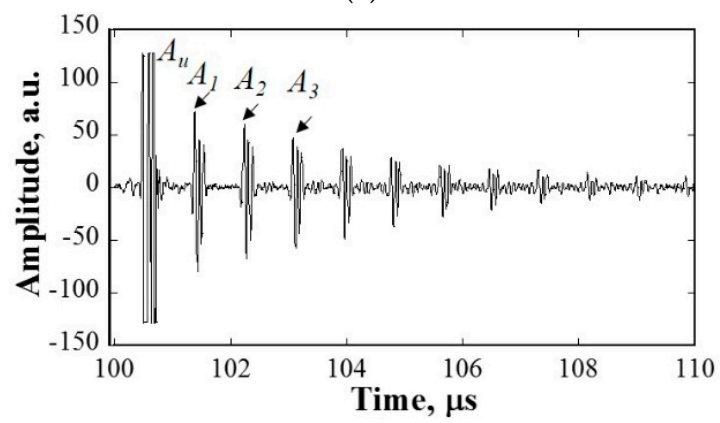

(c)

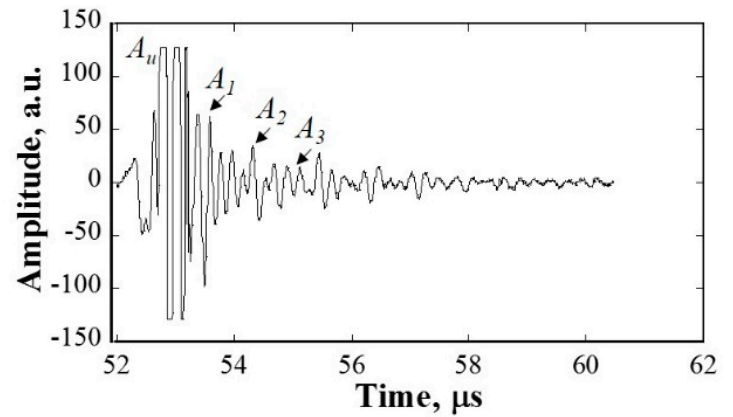

(b)

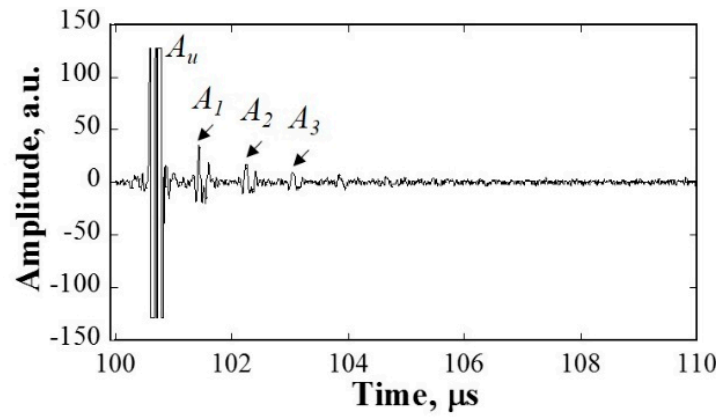

(d)

Figure 9. Cont. 


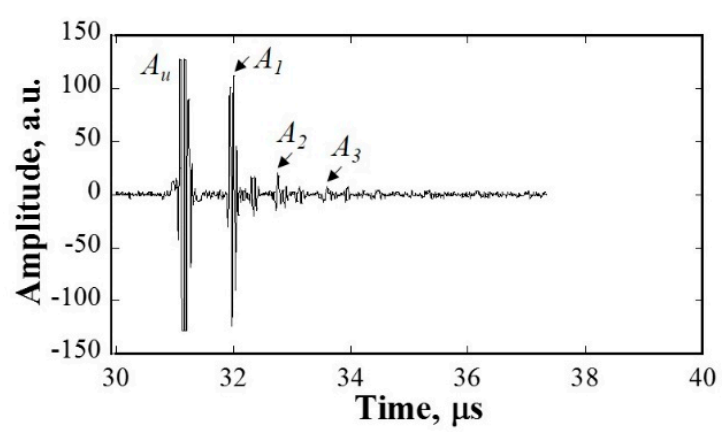

(e)

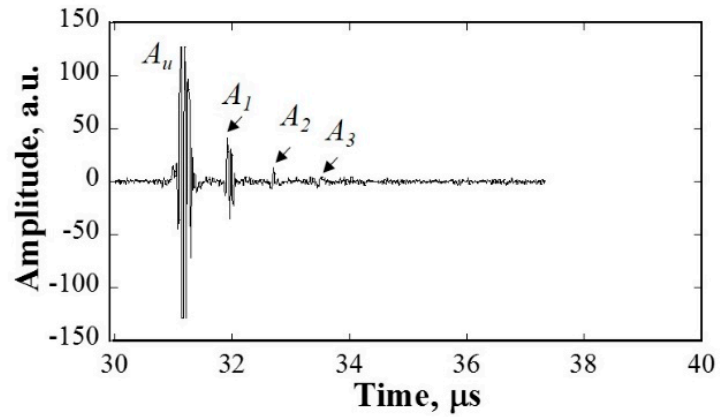

(f)

Figure 9. Examples of waveforms received from various samples at different frequencies: (a) as received at $5 \mathrm{MHz}$; (b) $1373 \mathrm{~K}$ at $5 \mathrm{MHz}$; (c) as received at $10 \mathrm{MHz}$; (d) $1373 \mathrm{~K}$ at $10 \mathrm{MHz}$; (e) as received at $20 \mathrm{MHz}$; (f) $1373 \mathrm{~K}$ at $20 \mathrm{MHz}$.

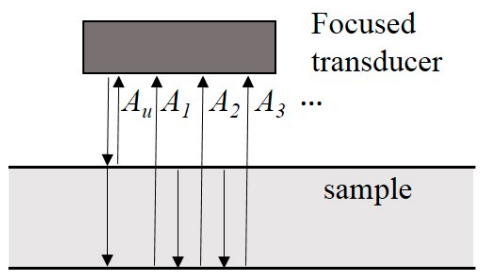

Figure 10. Schematic drawing of wave propagation.

The amplitude of the signals reflected from the upper surface $\left(A_{u}\right)$ could be considered the same because the amplitude of the incident waves and the distance from the focused transducer to the upper surface of specimen are the same. However, the amplitude of the signals reflected from the bottom surface $\left(A_{1}, A_{2}\right.$, and $\left.A_{3}\right)$ show significant differences owing to variations in the grain sizes of the specimens.

Therefore, the scattering of ultrasonic waves due to grains is different in the cases of different heat-treated samples. The attenuation coefficient due to grain scattering can be calculated from the amplitude of the three first back-wall signals, which can be expressed as follows [26]:

$$
\alpha_{E}=\frac{1}{2 t} \ln \left(R_{L}{ }^{2} \frac{\left|A_{n}-A_{n+1}\right|}{\left|A_{n+1}-A_{n+2}\right|}\right),
$$

where $\alpha_{E}$ is the attenuation coefficient calculated from experimental results; $R_{L}$ is the longitudinal wave reflection coefficient at the steel-water interface; and $t$ is the time of ultrasonic waves propagating between the upper and the bottom surfaces of the specimen.

The relationship between the attenuation coefficient and the frequency of the incident waveforms is shown in Figure 11. The attenuation coefficient increases with increasing frequency for a given grain size. Figure 12 shows the attenuation coefficient as a function of grain size at $20 \mathrm{MHz}$, and the attenuation increases with grain size at a given frequency. 


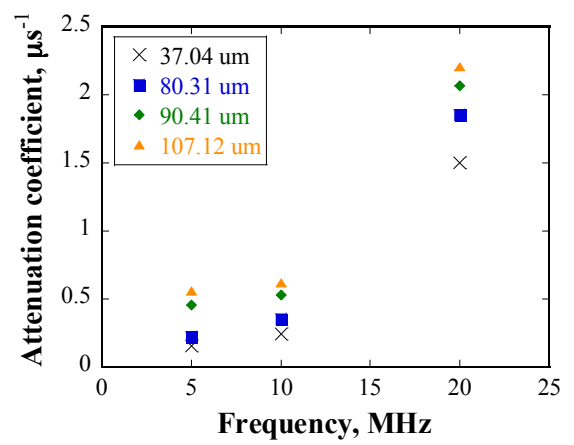

Figure 11. Relationship between attenuation coefficient and frequency for various mean grain sizes.

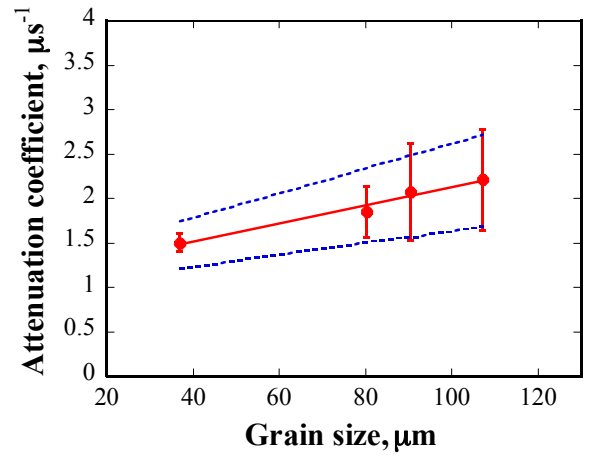

Figure 12. Relationship between attenuation coefficient and grain size (at $20 \mathrm{MHz}$ ); the two dashed lines indicate the boundary of standard deviation of the linear fitting curve.

\subsection{Numerical Simulation Results}

Figure 13 shows the displacement profile of the source node with a frequency of $80 \mathrm{MHz}$ in the numerical simulations. Models A and B were used for the calculation. Figure 14 shows examples of the normalized displacement profiles of the received waveforms in the cases of the same $E$ and a different $E$. Two signals were received, the first signal corresponds to the arrival of longitudinal waves and the second one corresponds to the arrival of transverse waves. Provided that the grains have the same $E$, the amplitudes of the received waveforms are independent of the grain size and are the same. If the grains are assigned $E$ with a normal distribution, the amplitudes of the received waveforms are dependent on grain size. The wavelength of the ultrasonic waves was approximately $74 \mu \mathrm{m}$, which is considerably smaller than the grain sizes. Therefore, geometric scattering occurred, and the amplitude of received waveforms is proportional to the grain size. Furthermore, the arrival time of the waves was delayed, because the wave propagation velocity in material changed, owing to the variation of $E$.

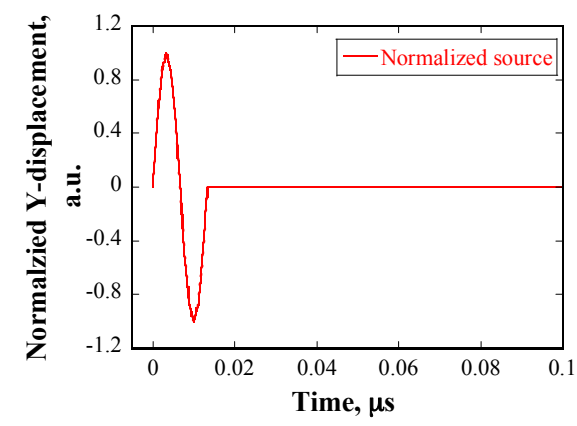

Figure 13. Normalized displacement profile of the source with a frequency of $80 \mathrm{MHz}$. 


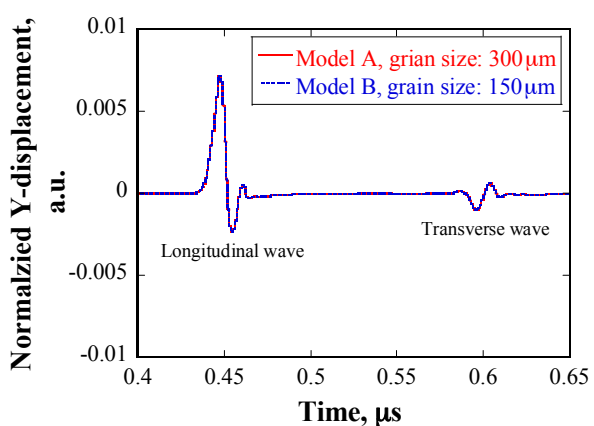

(a)

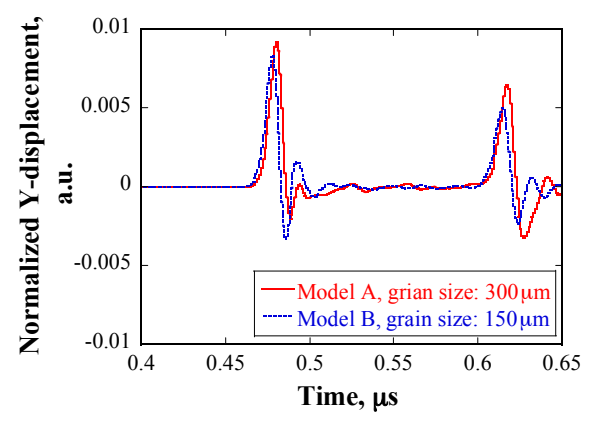

(b)

Figure 14. Normalized displacement profile of received waveforms at $80 \mathrm{MHz}$ in cases of the same $E$ and a different $E$ : (a) in case of the same $E ;(\mathbf{b})$ in case of a different $E$.

\section{Discussions}

\subsection{Validation of Scattering Regime for Experimental Results}

From Figure 11, it can be seen that the attenuation has a power law dependence on the incident frequency, with an index ranging from 0.99 to 1.62 for all specimens. These values are close to the power law index of two in the stochastic scattering regime, as shown in Table 1.

The frequency of the incident waveforms in the experiments ranges from $5-20 \mathrm{MHz}$; thus, the wavelength of the ultrasonic waves ranges from $285-1140 \mu \mathrm{m}$. As mentioned previously, the grain sizes were measured from 2D cross sections. However, the grain sizes used in the ultrasonic scattering theory should be three-dimensional (3D) values. Therefore, it is necessary to calibrate the measured 2D average grain size to an equivalent $3 \mathrm{D}$ value $D_{3}$. Here, a factor of two was adopted to multiply the $2 \mathrm{D}$ average grain size [27]. As a result, $2 \pi D_{3}$ ranges from $464-1346 \mu \mathrm{m}$, the magnitude of which is similar to that of the wavelengths of the incident waveforms in the experiments. Therefore, the scattering due to grains in the present study is located in the stochastic scattering regime.

Furthermore, the attenuation coefficient, $\alpha_{E}$, is linearly proportional to the average grain size, $\bar{D}$, as shown in Figure 12. The best fitting yielded the following linear relationship:

$$
\alpha_{E}=A \cdot \bar{D}+B
$$

where, $A=0.0102 ; B=1.1027 ; R^{2}=0.97$. The standard deviation of the factors $A$ and $B$ can be calculated using the following expression [25]:

$$
\begin{gathered}
\sigma_{A}=\sigma_{\alpha_{E}} \sqrt{\frac{\sum \bar{D}_{i}^{2}}{\triangle}}, \\
\sigma_{B}=\sigma_{\alpha_{E}} \sqrt{\frac{N}{\triangle}}, \\
\sigma_{\alpha_{E}}=\sqrt{\frac{1}{N-2} \sum_{i=1}^{N}\left(\alpha_{E, i}-A \cdot \bar{D}_{i}-B\right)^{2}}, \\
\triangle=N \sum \bar{D}_{i}^{2}-\left(\sum \bar{D}_{i}\right)^{2},
\end{gathered}
$$

where, $\sigma_{A}$ and $\sigma_{B}$ are the standard deviations of factors $A$ and $B$, respectively; $\sigma_{\alpha_{E}}$ is the uncertainty of the measured $\alpha_{E}$; and $\triangle$ is the denominator. The results show that $\sigma_{A}=0.003575$ and $\sigma_{B}=0.1325$. Accordingly, the value of factor $A$ falls within $0.0102 \pm 0.003575$, while the value of factor $B$ falls within $1.1027 \pm 0.1325$. The two dashed lines in Figure 12 indicate the boundary of the standard deviation of 
the linear fitting curve. It can be seen that most of the experimental data are located in the range of the two dashed lines.

Another issue of concern is that the deviation of the attenuation coefficient seems large. As presented above, although the grain size distributions are in good agreement with the log-normal distribution, there are several extremely large or small grains. In the Rayleigh and stochastic scattering regimes, the attenuation coefficient has a monotonous correlation with the grain size. As a result, it is easy to understand that the grains with abnormal sizes lead to extremely large or small attenuation coefficients, resulting in a large deviation of the attenuation coefficient. The effects of grains of extraordinary size on the attenuation coefficient will be discussed in detail in Section sect:sec6dot3-materials-10-00753.

To investigate whether the attenuation coefficient data has a statistical meaning, an analysis of variance (ANOVA) of the four groups of measured attenuation coefficient data (shown in Figure 12) was performed. The results of this analysis showed that the F-factor of ANOVA was 2.67983, which is larger than the threshold value of 2.46181 , with a confidence level of $90 \%$, under the present experimental conditions. The results imply that the differences between the groups of attenuation coefficient data are significant, with a confidence level of $90 \%$, and thus, it is reasonable to consider that the attenuation coefficient as a function of grain size has physical meaning.

Therefore, it can be concluded that the correlation between the attenuation coefficient and the mean grain size is linear in the present study, which fits well with the theory of the stochastic scattering regime. The experiment provides the possibility to quantitatively evaluate the grain size considering the linear relationship between the attenuation coefficient due to grain scattering and grain size.

\subsection{Comparison of Ultrasonic Scattering between Numerical Simulation and Experimental Results}

Figure 15 shows the normalized displacement as a function of source frequency at the receiver position, as obtained from models $A$ and $B$, respectively. When the source frequency is less than $5 \mathrm{MHz}$, the amplitude of displacement in the case of $300 \mu \mathrm{m}$ is larger than that in the case of $150 \mu \mathrm{m}$; as the source frequency increases, the displacement amplitude decreases to a greater extent in the case of $300 \mu \mathrm{m}$ than that in the case of $150 \mu \mathrm{m}$. Furthermore, the dependency of the displacement amplitude on frequency can be divided into three regions: in the region where the frequency is below $3 \mathrm{MHz}$, the displacement declines rapidly; when the frequency is between $4-30 \mathrm{MHz}$, the slope tends to be smaller; and, when the frequency is above $30 \mathrm{MHz}$, the slope tends to be larger again, but not as steep as that in the first region.

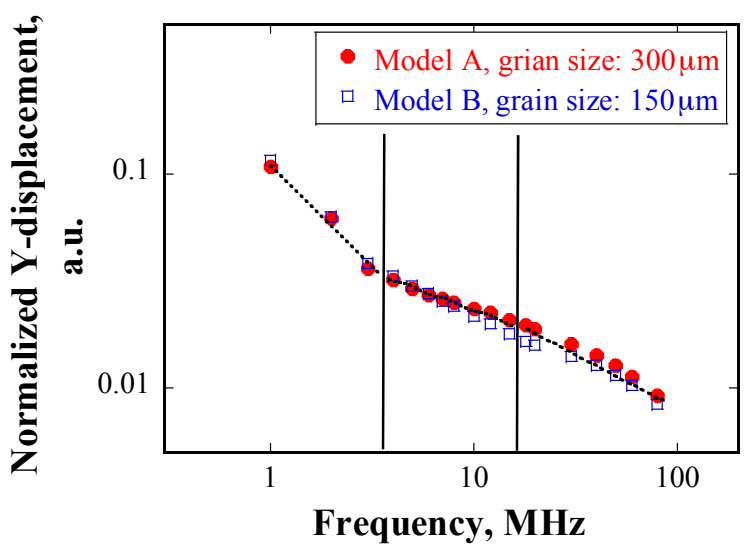

Figure 15. Displacement as a function of source frequency at detection position; obtained using models A and B.

The phenomenon presented above is closely related to the scattering of ultrasonic waves by the grains. To calculate the absolute attenuation coefficient caused by grain scattering, it is necessary to eliminate the effects of absorption and spreading on results. To achieve this objective, the displacement 
amplitudes obtained using model B with the same $E$ were used in the calculation. This assumes that the displacement amplitude at the receiver is the same in the case of the same $E$ and that it is different in the case of a different $E$. Then, the displacement amplitude caused merely by scattering, $A_{S}$, can be expressed as follows:

$$
A_{S}=\left|A_{\text {same }}-A_{\text {different }}\right|
$$

Substituting Equation (15) into Equation (2), we get:

$$
\left|A_{\text {same }}-A_{\text {different }}\right|=A_{0} \cdot e^{-\alpha^{\prime \prime} \cdot t}
$$

Then, the attenuation coefficient can be calculated using the following equation:

$$
\alpha^{\prime \prime}=-\ln \left(\frac{\left|A_{\text {same }}-A_{\text {different }}\right|}{A_{0}}\right) / \mathrm{t},
$$

where $\alpha^{\prime \prime}$ is the absolute attenuation coefficient owing to grain scattering obtained from the numerical simulation results.

$\alpha^{\prime \prime}$ was calculated according to Equation (17), and it is plotted as a function of frequency in Figure 16. The grain size is $300 \mu \mathrm{m}$. The dependency of the attenuation coefficient on frequency can be divided into three regions. First, if the wavelength is much higher than the grain size, the correlation can be expressed as follows:

$$
\alpha^{\prime \prime}=c f^{d}
$$

Second, if the wavelength is similar to the grain size, the correlation is given as follows:

$$
\alpha^{\prime \prime}=m f^{n}
$$

Finally, if the wavelength is much smaller than the grain size, the attenuation coefficient is independent of the frequency. Here $c, d, m$, and $n$ are constants related to the material property. The best-fitting result in Figure 16 shows that $c$ equals 17.11, $d$ equals $0.12, m$ equals 18.76, and $n$ equals 0.059 , in the present study.

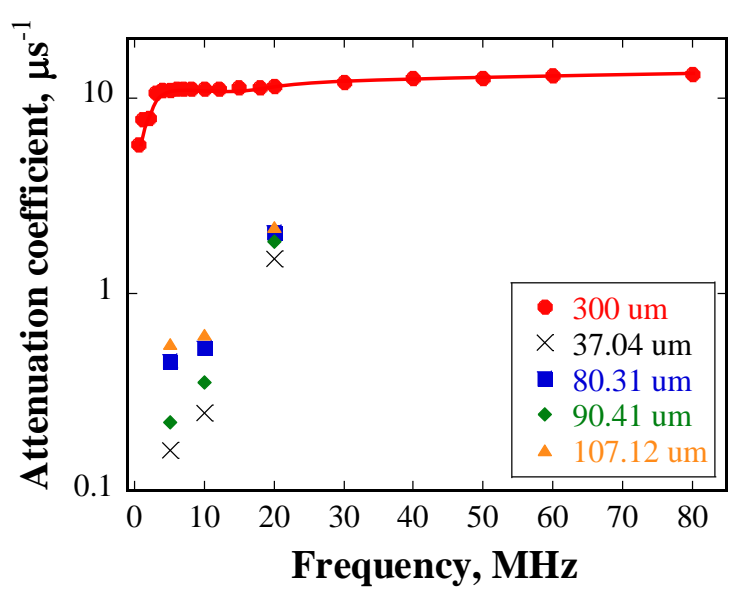

Figure 16. Attenuation coefficient as a function of frequency for various mean grain sizes.

Wave scattering can also be divided into three regimes. In the first and the second regimes, the attenuation coefficient is proportional to frequency and shows a power law relationship with frequency. The power law indexes are smaller in the 2D case than those in the 3D case, as listed in Table 1. However, the ratios of the power law of the first type to that of the second type are almost the same in both cases, which is a factor of two. In the last regime, when the wavelength is considerably 
smaller than the grain size, the attenuation coefficient is insensitive to the frequency, which indicates geometrical scattering.

The experimental data are plotted together for comparison in Figure 15. In case of the frequency range of $5 \mathrm{MHz}$ to $20 \mathrm{MHz}$, grain scattering in both the numerical simulations and the experiments is located in the stochastic regime. However, given that the grain size in the numerical simulation is considerably larger than that in the experiments, the dependency of attenuation coefficients on grain size is not linear. One possible reason is that the inhomogeneity of grain size distribution in the heat-treated samples results in a large scatter of the calculated attenuation coefficients. Taking the specimen heat-treated at $1473 \mathrm{~K}$ with a mean grain size of $107.12 \mu \mathrm{m}$ as an example, although the calculated average attenuation coefficient is approximately 2.1 at $20 \mathrm{MHz}$, the maximum attenuation coefficient can reach approximately 2.8, owing to deviation in the results. The attenuation coefficient obtained from numerical simulation in the case of grain size of $300 \mu \mathrm{m}$ at $20 \mathrm{MHz}$ is approximately 11.4, which is about four times that in the experimental data. From the above discussion, it can be deduced that the numerical results roughly coincide with the experimental ones in the stochastic scattering regime. The effect of grain size distribution on scattering will be further discussed in the next sub-section. Another factor that contributes to the differences between the attenuation coefficients obtained in the numerical simulations and the experiments is the difference in the grains geometry in the two cases.

\subsection{Scattering of Ultrasonic Wave Due to Random Grains}

Figure 17 shows the attenuation coefficient as a function of source frequency, as obtained using models $\mathrm{C}$ and $\mathrm{D}$. The correlation between the attenuation coefficient and frequency can also be divided into three regions; namely, Rayleigh scattering (below $5 \mathrm{MHz}$ ), stochastic scattering (approximately $5-30 \mathrm{MHz}$ ), and geometric scattering (above $30 \mathrm{MHz}$ ). In the case of Model $\mathrm{C}$, the attenuation coefficient is proportional to frequency in the Rayleigh and the stochastic regimes, and the attenuation coefficient remains constant and independent of frequency in the geometric regime. However, the attenuation coefficients are inversely proportional to frequency in the stochastic regime in the case of Model D. Moreover, the results indicate that the attenuation coefficient is inversely proportional to grain size in the Rayleigh and the stochastic regime, whereas it is proportional to grain size in the geometric regime. Such relationships are contrary to the scattering theory.

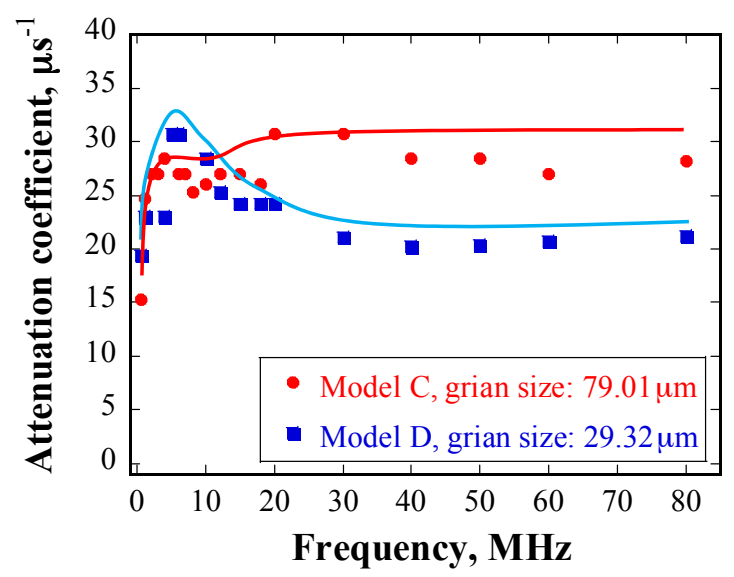

Figure 17. Attenuation coefficient as a function of source frequency at detection position, as obtained using models $\mathrm{C}$ and D.

This might be caused by the considerably inhomogeneous grain distribution in Model D. Figure 5b shows that a few grains in the wave propagation path in Model D are very large, while other grains are considerably small. By contrast, the grain sizes are relatively uniform in the case of Model C, as shown in Figure 5a. Actually, a grain size distribution analysis of Model D shows that over $60 \%$ of 
the grains are smaller than $25 \mu \mathrm{m}$, while over $0.3 \%$ of the grains are larger than $380 \mu \mathrm{m}$. It has been reported that in the Rayleigh scattering regime, the effect of one large grain on wave scattering is greater than the sum of the effects of many small grains in the same volume [17]. It is then reasonable to assume that this holds in the stochastic scattering regime and the geometric scattering regimes as well. It is considered that the scattering behavior of Model D is affected strongly by the extraordinarily large grains, which are considerably larger than the average grain size in Model C. This explains why the dependency of the attenuation coefficient on mean grain size shown in Figure 16 is contrary to expectations according to the scattering theory.

Regarding the descending relationship between the attenuation coefficient and frequency in the stochastic regime for Model D, the inhomogeneous grain size distribution may also play a dominant role. As the frequency of ultrasonic wave increases, their wavelength decreases. The wavelength tends gradually towards the size of large grains and then to that of small grains, which represent entry into and exit from the stochastic scattering regime, respectively. According to the stochastic scattering theory, the attenuation coefficient is proportional to both grain size and frequency. If grain size distribution was homogenous, it would be easy to understand that the attenuation coefficient is dependent solely on the frequency. Supposing that the grain size differs largely, it is also reasonable to assume that the large grains determine the attenuation coefficient at the beginning of the stochastic scattering regime, whereas the relatively smaller grains determine the attenuation coefficient at the end of the stochastic scattering regime. Therefore, although the wave frequency increases from the beginning to the end of the stochastic scattering regime, the larger attenuation coefficient obtained at the beginning can be attributed to a considerably larger grain size.

\section{Conclusions}

To understand the effects of grain size on the propagation behavior of ultrasonic wave in type 316L stainless steel, experimental and numerical studies were carried out. We focused on the attenuation coefficient of ultrasonic waves in this study, and the results obtained are summarized below:

(1) The attenuation coefficient of ultrasonic waves propagating in 316L SS with log-normal grain size distribution follows a power law with frequency, with an average index of 1.31 and a linear relationship with the average grain size. Given that the wavelength of ultrasonic waves ranges from $285-1140 \mu \mathrm{m}$, which is similar to the grain sizes range of $464-1346 \mu \mathrm{m}$, ultrasonic wave scattering falls into the stochastic scattering regime, and the correlation between the attenuation coefficient and mean grain size shows good agreement with the wave scattering theory.

(2) A numerical model approach was proposed to investigate the interactions between grains of various sizes and ultrasonic waves of a wide frequency range. The attenuation coefficient of ultrasonic waves due to scattering can be divided into three regimes according to the correlation between wavelength $(\lambda)$ and average grain size $(D)$ : when $\lambda>2 \pi D$ and $\lambda \approx 2 \pi D$, the relationship between the attenuation coefficient and frequency follows a power law, and the index of the power law is two times in the former case to the latter case; when $\lambda \ll D$, the attenuation coefficient is independent of frequency. These results are supported by the wave scattering theory.

(3) The attenuation coefficient obtained from the numerical results is approximately double relative to that obtained experimentally with a large error bar, which is attributed to the inhomogeneous distributions of grain size in the specimens. Localized grain size distribution in the ultrasonic wave path strongly affects the attenuation coefficient of ultrasonic waves.

(4) Although average grain size is an important parameter for determining the scattering behavior of ultrasonic wave, grains with extraordinarily large sizes are dominant. This dominance is reflected by the fact that compared to the attenuation coefficients calculated based on the mean grain size, the attenuation coefficients affected by extraordinarily large grains are considerably larger in the Rayleigh and the stochastic scattering regime, and considerably smaller in the 
geometric scattering regime. The boundaries of the different scattering regimes can be altered by the extraordinarily large grains.

This work provides an exact approach to quantitatively evaluate the grain size by calculating its relationship with the attenuation coefficient of ultrasonic waves. The basic knowledge obtained can be applied to monitor the material status of many sorts of industrial structures in the future, such as pipes, pressure vessels, and power plants.

Acknowledgments: The authors thank sincerely for Hideaki Ishii of Metal Technology Company for preparing the specimens and for measuring the grain sizes.

Author Contributions: Tao Wan and Masatoshi Futakawa conceived and designed the experiments and numerical simulations; Tao Wan and Takashi Naoe performed the experiments; Tao Wan and Takashi Wakui performed the numerical simulations; Tao Wan analyzed the data, and was responsible for writing the paper; Hironari Obayashi and Toshinobu Sasa provided useful discussions for the background.

Conflicts of Interest: The authors declare no conflict of interest.

\section{References}

1. Oigawa, H.; Tsujimoto, K.; Nishihara, K.; Sugawara, T.; Kurata, Y.; Takei, H.; Saito, S.; Sasa, T.; Obayashi, H. Role of ADS in the back-end of the fuel cycle strategies and associated design activities: The case of Japan. J. Nucl. Mater. 2011, 415, 229-236. [CrossRef]

2. Tsujimoto, K.; Oigawa, H.; Ouchi, N.; Kikuchi, K.; Kurata, Y.; Mizumoto, M.; Sasa, T.; Saito, S.; Nishihara, K.; Umeno, M.; et al. Research and development program on accelerator driven subcritical system in JAEA. J. Nucl. Sci. Technol. 2007, 44, 483-490. [CrossRef]

3. Sasa, T. Design of J-PARC transmutation experimental facility. Prog. Nucl. Energy 2015, 82, 64-68. [CrossRef]

4. Sasa, T.; Oigawa, H.; Tsujimoto, K.; Nishihara, K.; Kikuchi, K.; Kurata, Y.; Saito, S.; Futakawa, M.; Umeno, M.; Ouchi, N.; et al. Research and development on accelerator-driven transmutation system at JAERI. Nucl. Eng. Des. 2004, 230, 209-222. [CrossRef]

5. Obayashi, H.; Takei, H.; Wan, T.; Kogawa, H.; Iwamoto, H.; Sasa, T. TEF beam window design and evaluation of structural integrity. In Proceedings of the 2nd International Symposium on Science at J-PARC-Unlocking the Mysteries of Life, Matter and the Universe; JPS: Tokyo Japan, 2015. [CrossRef]

6. Zhang, J. A Review of steel corrosion by liquid lead and lead-bismuth. Corros. Sci. 2009, 51, 1207-1227. [CrossRef]

7. Ina, K.; Koizumi, H. Penetration of liquid metals into solid metals and liquid metal embrittlement. Mater. Sci. Eng. A 2004, 387, 390-394. [CrossRef]

8. Dai, Y.; Gavillet, D.; Restani, R. Stressed capsules of austenitic and martensitic steels irradiated in SINQ Target-4 in contact with liquid lead-bismuth eutectic. J. Nucl. Mater. 2008, 377, 225-231. [CrossRef]

9. Wan, T.; Naoe, T.; Futakawa, M. In-situ structural integrity evaluation for high-power pulsed spallation neutron source: Effects of cavitation damage on structural vibration. J. Nucl. Mater. 2016, 468, 321-330. [CrossRef]

10. Wan, T.; Naoe, T.; Wakui, T.; Futakawa, M.; Obayashi, H.; Sasa, T. Study on the evaluation of erosion damage by using laser ultrasonic integrated with a wavelet analysis technique. J. Phys. Conf. Ser. 2017, 842, 012010. [CrossRef]

11. Darbari, G.S.; Singh, R.P.; Verma, G.S. Ultrasonic attenuation in carbon steel and stainless steel at elevated temperatures. J. Appl. Phys. 1968, 39, 2238-2245. [CrossRef]

12. Sarpün, İ.H.; Kılıçkaya, M.S. Mean grain size determination in marbles by ultrasonic first backwall echo height measurements. NDT E Int. 2006, 39, 82-86. [CrossRef]

13. Abramov, O.V. Ultrasound in Liquid and Solid Metals; CRC Press, Inc.: Boca Raton, FL, USA, 1994; pp. 121-160.

14. Roth, W. Scattering of ultrasonic radiation in polycrystalline metals. J. Appl. Phys. 1948, 19, 901-910. [CrossRef]

15. Hirsekorn, S.; Van Andel, P.W.; Netzelmann, U. Ultrasonic methods to detect and evaluate damage in steel. Nondestr. Test. Eval. 1998, 15, 373-393. [CrossRef]

16. Papadakis, E.P. Rayleigh and stochastic scattering of ultrasonic waves in steel. J. Appl. Phys. 1963, 34, $265-269$. [CrossRef] 
17. Papadakis, E.P. From micrograph to grain-size distribution with ultrasonic applications. J. Appl. Phys. 1964, 35, 1586-1594. [CrossRef]

18. Smith, R.L. The effect of grain size distribution on the frequency dependence of the ultrasonic attenuation in polycrystalline materials. Ultrasonics 1982, 20, 211-214. [CrossRef]

19. Wan, T.; Naoe, T.; Wakui, T.; Futakawa, M.; Kawashima, K.; Maekawa, K. Pitting damage imaging by non-linear ultrasonic technique: Comparison between resonance and non-resonance modes. Int. J. Mater. Prod. Technol. 2013, 46, 141-153. [CrossRef]

20. Keywork User's Manual, version 5869; Livermore Software Technology Corporation: Livermore, CA, USA, 2014.

21. Kim, J.K.; Woo, J.; Na, W.B. Finite element simulation of two-point elastic wave excitation method for damage detection in concrete structures. Russ. J. Nondestr. Test. 2008, 44, 719-726. [CrossRef]

22. Hora, P.; Michálek, J. Numerical modeling of dispersion phenomena in thick plate. Exp. Stress Anal. 2000, 95-102.

23. Ritz, H.; Dawson, P.R. Sensitivity to grain discretization of the simulated crystal stress distributions in FCC polycrystals. Model. Simul. Mater. Sci. Eng. 2009, 17, 015001. [CrossRef]

24. Ogi, H.; Niho, H.; Hirao, M. Elastic-stiffness distribution on dual-phase stainless steel studied by resonance ultrasound microscopy. Acta Mater. 2006, 54, 4143-4148. [CrossRef]

25. Taylor, J.R. An Introduction to Error Analysis: The Study of Uncertainties in Physical Measurements, 2nd ed.; University Science Books: Sausalito, CA, USA, 1997; pp. 147-149 and pp. 186-188.

26. Bouda, A.B.; Lebaili, S.; Benchaala, A. Grain size influence on ultrasonic velocities and attenuation. NDT E Int. 2003, 36, 1-5. [CrossRef]

27. Stanke, F.E.; Kino, G.S. A unified theory for elastic wave propagation in polycrystalline materials. J. Acoust. Soc. Am. 1984, 75, 665-681. [CrossRef]

(C) 2017 by the authors. Licensee MDPI, Basel, Switzerland. This article is an open access article distributed under the terms and conditions of the Creative Commons Attribution (CC BY) license (http:// creativecommons.org/licenses/by/4.0/). 